\title{
FREQUENCY INVARIANT BEAMFORMING IN SUBBANDS
}

\author{
Wei Liu
}

\author{
Communications \& Signal Processing Group \\ Dept. of Electrical \& Electronic Engineering \\ Imperial College London, U.K.
}

Stephan Weiss
Communications Research Group

School of Electronics \& Computer Science

University of Southampton, U.K.

\begin{abstract}
In this paper, two subband implementations of a frequency invariant beamformer (FIB) are studied. In the first structure, the received array signals are split into subbands and an FIB is operated in each of the corresponding decimated subbands, with a potential of achieving a lower computational complexity. As the spatio-temporal distribution of the subband signals is different from the original fullband signal, a modified design method of the FIB is proposed. Based on the subband implementation, we then change the sensor spacings of different subband signals so that lower frequency bands have a larger spacing, which results in a class of FIBs with scaled aperture with improved performance in lower frequencies. Several design examples are given to show the performance of our new structures.
\end{abstract}

\section{INTRODUCTION}

In the past, broadband beamformers have been studied extensively due to their applications to sonar, radar and communications [1]. Amongst them is a class of arrays with frequency invariant beam patterns [2, 3]. Most recently, a new class of frequency-invariant broadband arrays, which exploits the Fourier transform relationship between the array's spatio-temporal distribution and its beam pattern, has been proposed [4]. Starting from the desired frequency-invariant beam pattern, by a series of substitutions a simple design method was found for frequency invariant beamforming design. This method can be applied to 1-D, 2-D and 3-D broadband arrays. A previously proposed frequency invariant linear array [5] can be regarded as a special case of this new class of arrays.

In this paper, we further exploit the potentials of this new class of arrays and present two subband implementations of it. In the first structure, the received array signals are split into subbands and a frequency invariant beam- former (FIB) is operated in each of the corresponding decimated subbands. When the spatio-temporal dimension of the fullband array becomes large enough, the subband implementation can provide a better performance at lower frequencies with a lower computational complexity, although the frequency invariant property on the whole is not as good as the fullband FIB due to the aliasing problem. As the spatio-temporal distribution of the subband signals is different from the original fullband signal, a modified design method of the FIB is proposed. In a refinement, we modify this subband FIB for use with nested arrays. Several design examples are given to show the performance of our new structures.

This paper is organised as follows. A brief review of FIBs is given in Section 2. We study its subband implementation in Section 3 and consider a nested array in Section 4. Design examples are given in Section 5, and conclusions drawn in Section 6.

\section{FREQUENCY INVARIANT BEAMFORMING}

Although the method proposed in [4] can be applied to 1-D, 2-D and 3-D broadband arrays, without loss of generality, we here focus only on the equispaced linear array.

Consider an equally spaced linear array with element spacing of $d_{x}$ and signal sampling period $T$. Its beam pattern $P(\omega, \theta)$ is given by

$$
P(\omega, \theta)=\sum_{m, n=-\infty}^{\infty} d[m, n] e^{-j m \frac{\omega \sin \theta}{c} d_{x}} e^{-j n \omega T},
$$

where $d[m, n]$ is the coefficient in the $n$-th position of the $m$-th sensor's tapped-delay line (TDL).

To avoid aliasing in both the spatial and temporal domains, $T$ should be half of the period of the maximum signal frequency of interest and $d_{x}$ half of its wavelength $\lambda_{\max }$. Thus we have $d_{x}=\frac{\lambda_{\max }}{2}=c T$ and $\omega T=\Omega$, where $\Omega$ is 


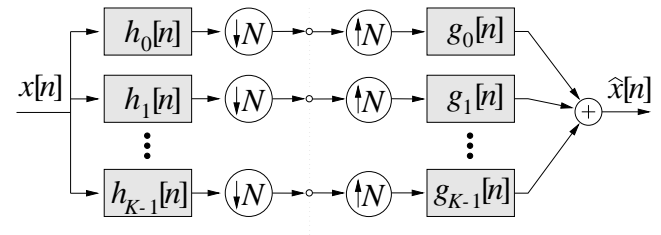

analysis filter bank $\quad$ synthesis filter bank

Fig. 1: A general structure of a $K$-channel filter bank with a decimation factor of $N$.

the normalised angular frequency. Therefore, (1) can be rewritten as

$$
P(\Omega, \theta)=\sum_{m, n=-\infty}^{\infty} d[m, n] e^{-j m \Omega \sin \theta} e^{-j n \Omega} .
$$

Substituting $\Omega_{1}=\Omega \sin \theta$ and $\Omega_{2}=\Omega$ into (2) yields

$$
P\left(\Omega_{1}, \Omega_{2}\right)=\sum_{m, n=-\infty}^{\infty} d[m, n] e^{-j m \Omega_{1}} e^{-j n \Omega_{2}} .
$$

As the spatio-temporal spectrum of the impinging signal lies on the line $\Omega_{1}=\Omega_{2} \sin \theta$, a method can be developed to obtain a frequency invariant beam pattern by following the design below.

Step 1. From the desired beam pattern $P(\sin \theta)$ we derive the frequency response of a 1 -D filter $F(\hat{\Omega})$, which is periodic with $2 \pi$, defined over one period as

$$
F(\hat{\Omega})=P(\hat{\Omega} / \pi) \quad \text { for } \quad \hat{\Omega} \in[-\pi ; \pi) .
$$

Step 2. With the substitution $\hat{\Omega}=\frac{\Omega_{1}}{\Omega_{2}} \pi$, for $\left(\Omega_{1}, \Omega_{2}\right) \in$ $[-\pi ; \pi)$ we have

$$
P\left(\Omega_{1}, \Omega_{2}\right)=\left\{\begin{array}{ll}
F\left(\left(\Omega_{1} / \Omega_{2}\right) \pi\right) & \text { for } \Omega_{2} \neq 0 \\
a\left(\Omega_{1}\right) & \text { for } \Omega_{2}=0
\end{array},\right.
$$

where $a\left(\Omega_{1}\right)$ is an arbitrary function with finite values. Note that $P\left(\Omega_{1}, \Omega_{2}\right)$ is a function with a period of $2 \pi$.

Step 3. Applying a 2-D inverse Fourier transform to $P\left(\Omega_{1}, \Omega_{2}\right)$ results in an infinite support of $d[m, n]$. As it is difficult to obtain result analytically, we can apply the 2 -D inverse DFT as an approximation by sampling $P\left(\Omega_{1}, \Omega_{2}\right)$. In either case, the resulting $d[m, n]$ needs to be delayed along the $n$ axis for reasons of causality and to be truncated according to the number of sensors and the TDL length.

\section{SUBBAND IMPLEMENTATION}

Fig. 1 shows the general structure of a $K$-channel filter bank with a decimation factor $N$. For the subband implementation of the FIB, each of the received array signals $x_{m}[n]$,

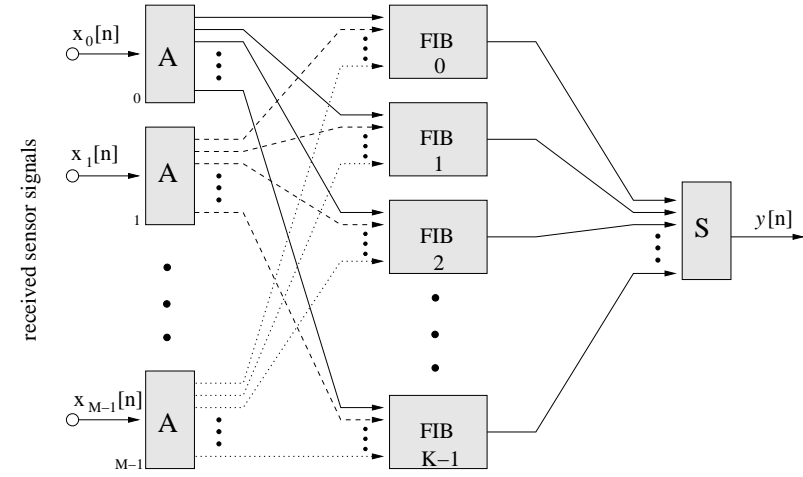

Fig. 2: Subband implementation of the frequency invariant beamformer.

$m=0,1, \cdots, M-1$, is split into $K$ subbands by an analysis filter bank and the corresponding subband signals form $K$ sets of subband arrays. An FIB is operated in each of the subband arrays and the processed subband signals are then combined together by a synthesis filter bank into the fullband output. The structure is shown in Fig. 2, where the blocks labeled "A" are the analysis filter banks and the block labeled " $\mathrm{B}$ " is the synthesis filter bank.

The advantage of this subband implementation is similar to the subband implementation of adaptive filters [6], i.e. due to the subband decomposition and decimation, the length of each of the TDLs of the subband frequency invariant beamformer can be shorter than the original fullband beamformer to achieve a similar performance, in accordance with the sampling rate reduction by a factor of $N<K$. This can be explained by the fact that $N$ subband FIBs are trying to model the original fullband beamformer to have the same spatio-temporal filtering effect.

Since the subband FIBs have a shorter length and operate at a lower rate, the computational complexity of the whole system can be much lower than the fullband implementation, when the fullband beamformer's TDLs are sufficiently long to render the additional filter bank operations negligible.

For the design of the subband beamformer, because the normalised signal angular frequency $\tilde{\Omega}$ in decimated subbands has changed after decimation, we need to modify the method in Sec. 2 to fit the new scenario.

For the design of the $i$-th subband beamformer, at first, we need to find the relationship $\tilde{\Omega}=S_{i}(\Omega)$ between the decimated subband $\tilde{\Omega}$ and the fullband $\Omega$. Suppose the spectrum of the input signal to the analysis filter bank is $X\left(e^{j \Omega}\right)$, then the output spectrum $Y_{i}\left(e^{j \tilde{\Omega}}\right)$ of the $i$-th anal- 
ysis filter after decimation is given by [7]

$$
Y_{i}\left(e^{j \tilde{\Omega}}\right)=\frac{1}{N} \sum_{p=0}^{N-1} X\left(e^{j\left(\frac{\tilde{\Omega}-2 p \pi}{N}\right)}\right) .
$$

We can find each $\tilde{\Omega}=S_{i}(\Omega)$ according to this equation.

For the $i$-th subband beamformer, its response to the decimated subband input signal can be written as

$$
\tilde{P}_{i}(\tilde{\Omega}, \theta)=\sum_{m, n=-\infty}^{\infty} d_{i}[m, n] e^{-j m \Omega \sin \theta} e^{-j n \tilde{\Omega}},
$$

where $d_{i}[m, n]$ is the coefficients of the $i$-th subband beamformer. We use the same phase difference $e^{-j \Omega \sin \theta}$ between adjacent sensors as that of the fullband beamformer in (2), because it does not change after decimation. For its response to the original fullband input signal, we have

$$
\tilde{P}_{i}\left(S_{i}(\Omega), \theta\right)=\sum_{m, n=-\infty}^{\infty} d_{i}[m, n] e^{-j m \Omega \sin \theta} e^{-j n S_{i}(\Omega)} .
$$

By substituting $\Omega_{1}=\Omega \sin \theta$ and $\tilde{\Omega}_{2}=\tilde{\Omega}$ into (7), we have

$$
\tilde{P}_{i}\left(\Omega_{1}, \tilde{\Omega}_{2}\right)=\sum_{m, n=-\infty}^{\infty} d_{i}[m, n] e^{-j m \Omega_{1}} e^{-j n \tilde{\Omega}_{2}} .
$$

Thus, we can obtain $d_{i}[m, n]$ by applying the inverse Fourier transform to the desired subband response $\tilde{P}_{i}\left(\Omega_{1}, \tilde{\Omega}_{2}\right)$. As $\tilde{\Omega}=S_{i}(\Omega)$ and $\Omega_{2}=\Omega$, we also have $\tilde{\Omega}_{2}=S_{i}\left(\Omega_{2}\right)$, then

$$
\tilde{P}_{i}\left(\Omega_{1}, S_{i}\left(\Omega_{2}\right)\right)=P\left(\Omega_{1}, \Omega_{2}\right)
$$

for the $i$-th subband.

From the discussion above, we obtain a modified method applicable to the subband beamformer using the inverse DFT.

Suppose the dimension of the $i$-th subband beamformer is $M_{i} \times J_{i}$,where $M_{i}$ is the sensor number and $J_{i}$ the TDL length. We first obtain $P\left(\Omega_{1}, \Omega_{2}\right)$ from the fullband beamformer design method. Then we uniformly sample $\Omega_{1}$ and $\tilde{\Omega}_{2}$ in $(-\pi ; \pi]$ with $M_{\max } \times J_{\max }$ points, where $M_{\max }>=$ $M_{i}$ and $J_{\max }>=J_{i}$. We calculate $\tilde{P}_{i}\left(\Omega_{1}, \tilde{\Omega}_{2}\right)$ on these points according to $(10)$. However, when the subband aliasing after decimation is too large, one value of $\tilde{\Omega}_{2}(\tilde{\Omega})$ will correspond to several different values of $\Omega_{2}(\Omega)$, where the $i$-th subband signal before decimation is not zero. Thus we will not be able to get unique values $\tilde{P}_{i}\left(\Omega_{1}, \tilde{\Omega}_{2}\right)$ on those sampling points. To avoid this problem, we need to employ oversampled filter banks to suppress the aliasing effect to an acceptable level, such as the oversampled GDFT filter banks [8], where although one value of $\tilde{\Omega}_{2}(\tilde{\Omega})$ still corresponds to several different values of $\Omega_{2}(\Omega)$, which is decided by (6) and can not be changed, only on one value of
$\Omega_{2}(\Omega)$, the $i$-th subband signal before decimation is significantly large compared to the other corresponding values of $\Omega_{2}(\Omega)$ and we will take that value to calculate the unique $\tilde{P}_{i}\left(\Omega_{1}, \tilde{\Omega}_{2}\right)$. Applying the inverse DFT to the result, we then get $d_{i}[m, n]$ with a dimension $M_{\max } \times J_{\max }$, which needs to be shifted and truncated to fit the real dimension $M_{i} \times J_{i}$

\section{FREQUENCY INVARIANT BEAMFORMING - SCALED APERTURE}

The spatial resolution of a beamformer is reciprocally proportional to both the aperture $D$ of the sensor array and the frequency $\Omega$ of an impinging waveform [1], therefore, it is difficult to achieve a constant beamwidth for lower frequencies. Although the design of the FIB becomes very simple by the method proposed in [4] and a constant beamwidth is achieved over a very large bandwidth, it is still frequency variant for the lower frequency band. To extend the constant beamwidth property to a frequency as low as possible, we here propose the frequency invariant beamformer with scaled aperture.

The idea is similar to the one proposed in [9] and reflected in the structure shown in Fig. 3, depicting the exemplary case for $M=4$ sensors for each octave and beamformers operating in 4 uniformly split decimated subbands, with each having a bandwidth $\pi / 4$, whereby the array signals are drawn from a total of 8 nested sensors. For the 3 octave groups of subband FIBs, processor \#0 operates on the lowest band, processors \#1 form the second octave, and the remaining two processors are responsible for the highest octave band covered by two subbands. As indicated in Fig. 3, not all subbands are required for processing from each sensor.

As the array spacing is different for each octave of subband beamformers, we need to change the subband beamformer design procedure correspondingly. In Fig. 3, beamformers 3 and 2 have a standard spacing of $d=\frac{\lambda_{\max }}{2}$, so we can apply the previous design procedure directly; for beamformers 1 and 0 , they have a spacing of $\lambda_{\max }$ and $2 \lambda_{\max }$, respectively, then we must consider this difference in our design. Suppose the spacing of the subband beamformer is $\sigma$ times the standard spacing $\frac{\lambda_{\max }}{2}$, then its response to the original fullband input signal is

$$
\tilde{P}_{i}\left(S_{i}(\Omega), \theta\right)=\sum_{m, n=-\infty}^{\infty} d_{i}[m, n] e^{-j m \sigma \Omega \sin \theta} e^{-j n S_{i}(\Omega)}
$$

where $d_{i}[m, n]$ is the coefficients of the $i$-th subband beamformer. By substituting $\tilde{\Omega}_{1}=\sigma \Omega \sin \theta=\sigma \Omega_{1}$ and $\tilde{\Omega}_{2}=$ 


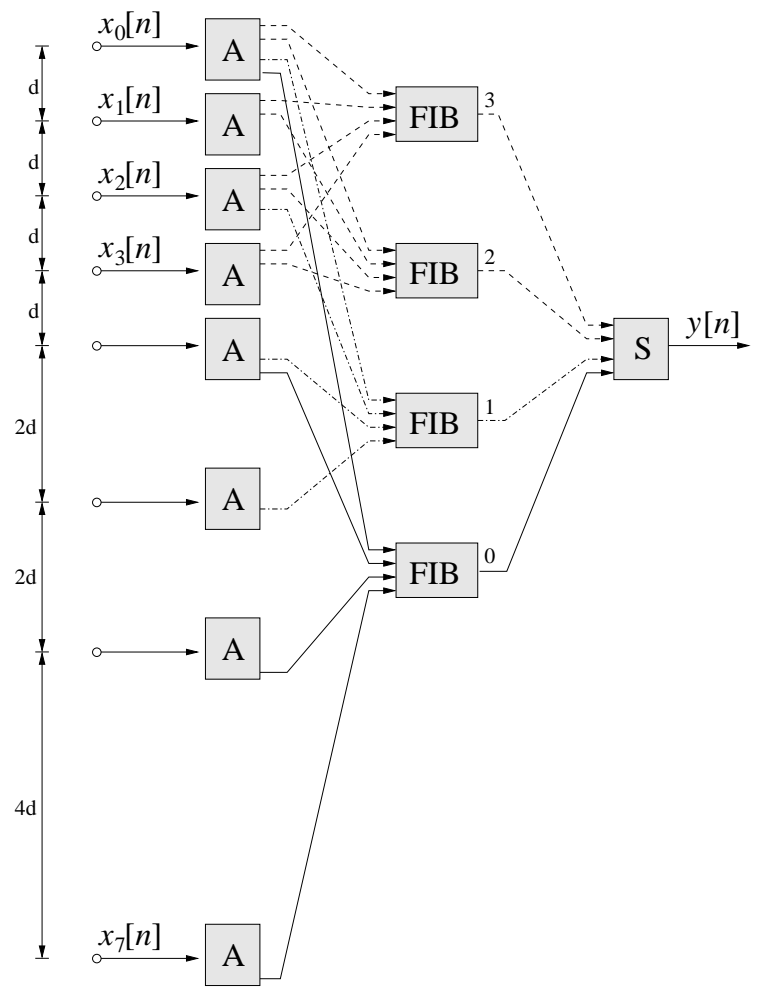

Fig. 3: Proposed beamforming structure with scaled array aperture for processors in various octave bands.

$S_{i}(\Omega)$ into (11), we have

$$
\tilde{P}_{i}\left(\tilde{\Omega}_{1}, \tilde{\Omega}_{2}\right)=\sum_{m, n=-\infty}^{\infty} d_{i}[m, n] e^{-j m \tilde{\Omega}_{1}} e^{-j n \tilde{\Omega}_{2}}
$$

The relationship between the subband beamformer response and the fullband beamformer response is given by

$$
\tilde{P}_{i}\left(\sigma \Omega_{1}, S_{i}\left(\Omega_{2}\right)\right)=P\left(\Omega_{1}, \Omega_{2}\right) .
$$

Then the design can be modified as the following.

First, we uniformly sample $\tilde{\Omega}_{1}$ and $\tilde{\Omega}_{2}$ in $(-\pi ; \pi]$ with $M_{\max } \times J_{\max }$ points. Then we obtain the response $\tilde{P}_{i}\left(\tilde{\Omega}_{1}, \tilde{\Omega}_{2}\right)$ on these points according to (13). With the inverse DFT, the temporal response $d_{i}[m, n]$ with a dimension $M_{\max } \times$ $J_{\text {max }}$ is then obtained. After proper shift and truncation, we get the final result with the real dimension $M_{i} \times J_{i}$.

\section{DESIGN EXAMPLE}

To show the improved performance by our method, we give one example for each of the implementations. The desired ideal response of our design is given by

$$
P(\sin \theta)=\frac{1}{5} \sum_{m=-2}^{2} e^{-j m \pi \sin \theta} .
$$

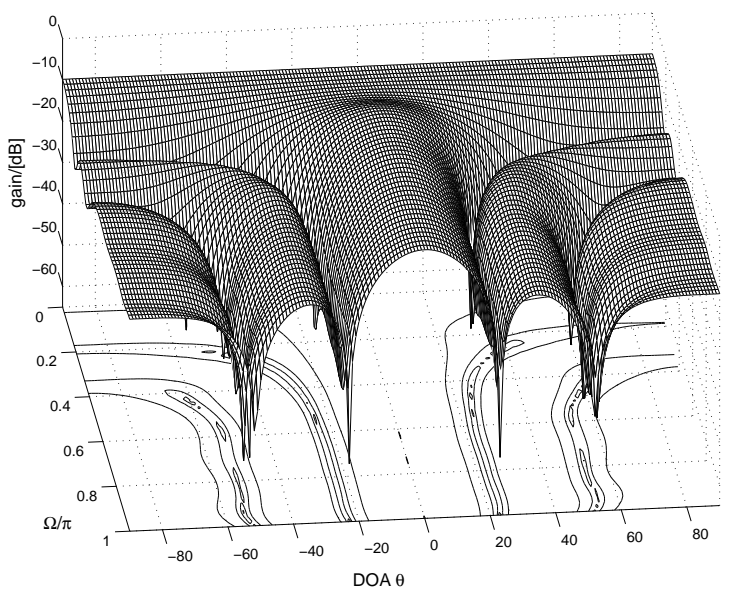

Fig. 4: The resultant beam pattern for the equispaced fullband linear array.

The fullband array has a dimension of $12 \times 16$ and the designed result is given in Fig. 4, which has a very good frequency invariant property for the range $[0.5 \pi ; \pi]$.

For the subband implementation, we employ the 6-channel oversampled GDFT filter banks with decimation ratio 4 [8]. Without loss of generality, we here only consider real-valued sensor signals, so that we only need to process half of the 6 subbands and in total there are 3 subband beamformers, each of which has a dimension of $12 \times 8$. The resultant beam pattern by our subband method is shown in Fig. 5, which is frequency invariant for frequencies even as low as $0.4 \pi$, although it seems that the frequency invariant property on the whole is not as good as the fullband example due to the aliasing problem. Note that the subband beamformer dimension is only half of the fullband beamformer, so the computational complexity of the beamformer part is reduced. But, in this case the computational complexity of the whole subband implementation is higher than the fullband case, as the extra complexity introduced by the filter banks part is higher than the complexity reduced. However, we can expect when the array dimension is larger enough, the subband implementation can have a lower computational complexity.

Next, for the array with scaled aperture, we employ the same filter banks. There are two octaves. For each of them, the subband beamformer has a dimension of $12 \times 8$. because of the increased complexity of the filter banks part, this scaled aperture system has a higher complexity than the subband implementation, but with a better performance, as shown in Fig. 6, where the frequency invariant property extends to frequencies lower than $0.2 \pi$. However, the fre- 


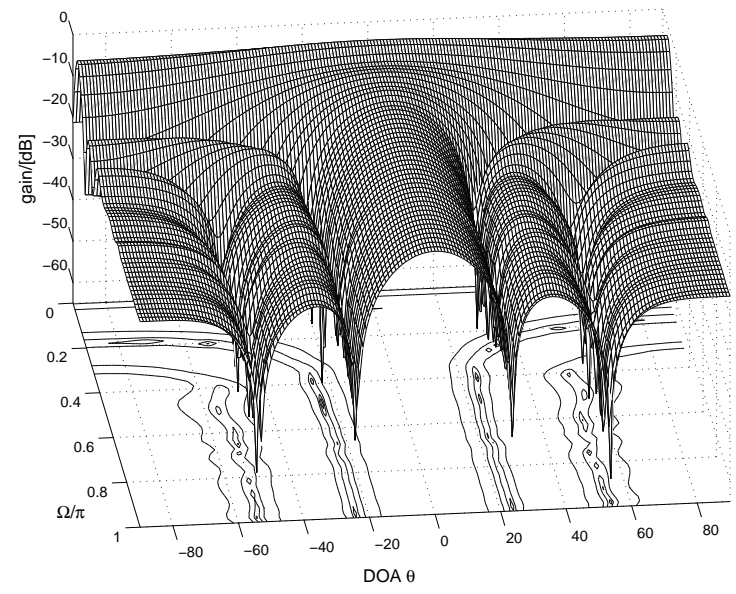

Fig. 5: The resultant beam pattern for the equispaced subband linear array.

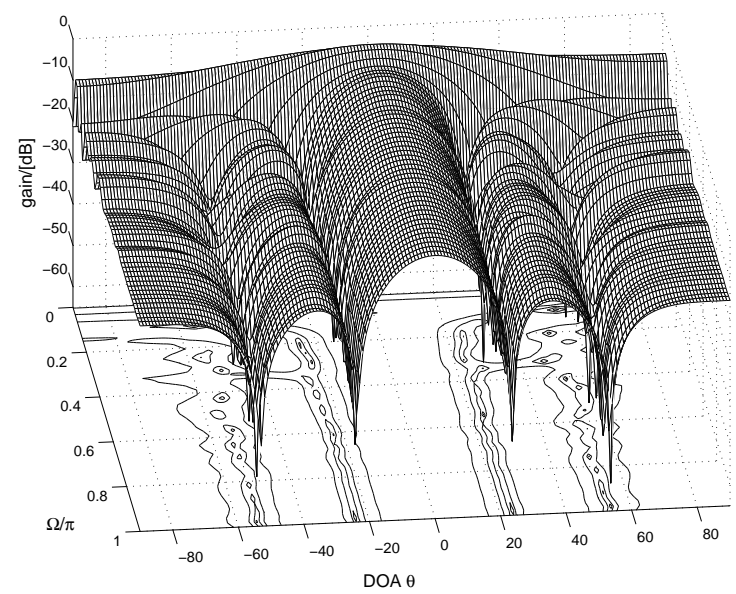

Fig. 6: The resultant beam pattern for the scaled aperture array.

quency invariant property on the whole is not as good as the above two examples.

\section{CONCLUSIONS}

Two subband implementations of the frequency invariant beamformer have been studied. In the first structure, the received array signals are split into subbands and a frequency invariant beamformer is operated in each of the corresponding decimated subbands. It has an improved performance at lower frequencies and a potential of achieving a lower computational complexity. For the second, we change the sensor spacings of different subband signals, resulting in a class of FIBs with scaled aperture with further improved performance at lower frequencies. However, there is also a problem with the two implementations, i.e. the degraded frequency invariant property on the whole, due to the aliasing problem of the filter banks.

\section{REFERENCES}

[1] H. L. Van Trees, Optimum Array Processing, Part IV of Detection, Estimation, and Modulation Theory, John Wiley \& Sons, Inc., New York, U.S.A., 2002.

[2] T. Chou, "Frequency-independent beamformer with low response error," in Proc. IEEE International Conference on Acoustics, Speech, and Signal Processing, Detroit, USA, May 1995, vol. 5, pp. 2995-2998.

[3] D. B. Ward, R. A. Kennedy, and R. C. Williamson, "Theory and design of broadband sensor arrays with frequency invariant far-field beam patterns," Journal of the Acoustic Society of America, vol. 97, no. 2, pp. 1023-1034, February 1995.

[4] W. Liu and S. Weiss, "A new class of broadband arrays with frequency invariant beam patterns," in Proc. IEEE International Conference on Acoustics, Speech, and Signal Processing, Montreal, Canada, May 2004, vol. 2, pp. $185-188$.

[5] T. Sekiguchi and Y. Karasawa, "Wideband Beamspace Adaptive Array Utilizing FIR Fan Filters for Multibeam Forming," IEEE Transactions on Signal Processing, vol. 48, no. 1, pp. 277-284, January 2000.

[6] A. Gilloire and M. Vetterli, "Adaptive Filtering in Subbands with Critical Sampling: Analysis, Experiments and Applications to Acoustic Echo Cancelation," IEEE Transactions on Signal Processing, vol. SP-40, no. 8, pp. 1862-1875, August 1992.

[7] P. P. Vaidyanathan, Multirate Systems and Filter Banks, Prentice Hall, Englewood Cliffs, 1993.

[8] M. Harteneck, S. Weiss, and R.W. Stewart, "Design of near perfect reconstruction oversampled filter banks for subband adaptive filters," IEEE Transactions on Circuits and Systems - II: Analog and Digital Signal Processing, vol. 46, pp. 1081-1085, August 1999.

[9] S. Weiss, R.W. Stewart, and W Liu, "A Broadband Adaptive Beamformer in Subbands with Scaled Aperture," in Proc. Asilomar Conference on Signals, Systems, and Computers, Monterey, CA, November 2002, pp. 1298-1302. 